\title{
On Synchronization of Chaotic Systems
}

\author{
Henk Nijmeijer \\ Department of Applied Mathematics \\ University of Twente, PO Box 217, 7500 AE Enschede \\ The Netherlands \\ phone $+31534893442 / 4893370$ \\ fax +31534340733 \\ e-mail: h.nijmeijer@math.utwente.nl
}

\begin{abstract}
This paper deals with the problem of synchronization, or observer design, of chaotic dynamical systems. It is argued that the complex nature of the transmitter dynamics may provide additional tools for finding a suitable observer. A number of characteristic examples illustrate the idea, and reveal some challenging open problems in this context.
\end{abstract}

\section{Introduction}

In recent years there has been considerable interest in the dynamics and control of systems exhibiting complex behavior. The number of papers related to this subject seems to grow at an almost exponential rate [8]. For an admittedly already 'dated' review of some of the prevailing research problems the reader may consult the seminal papers in [23].

The purpose of the present note is first to revisit the concept of synchronization from a mathematical control theoretic perspective. More specifically we want to explore how the observer notion from (non)linear control theory links in with synchronization. Further results along this line are reported in [19].

Synchronization, as introduced by Pecora and Carroll $[5,24]$ has been studied from various angles. Often a master-slave formalism is taken, $[5,24,12,6,25,10,17]$ and [7]. Given a particular dynamical system, the master, together with an identical (sub)system, the slave, the aim is to synchronize to the master system the complete response of the slave system, by driving the latter with a (scalar) signal derived from the master system. In this context synchronization is often considered to be a remarkable property when the master dynamics are chaotic and thus sensitive to initial condition variations. A promising application in secure communication suggested in [9] uses such a chaotic master dynamics to mask a message and a synchronized slave system to recover the message.

The above master-slave viewpoint leaves some ambiguity as to what the actual slave system should be, given the master system. A naive, but often realistic approach, would be to consider the master dynamics (transmitter) as transmitting a signal to the slave dynamics (receiver) and the receiver is requested to recover the full state trajectory of the transmitter. The problem is of course only interesting if the signal received is not equal to the full state. In this situation the receiver has in principle the freedom to build any dynamical system. The receiver system could be a copy of the master system, but it need not be. The real requirement is that given the received signal the receiver dynamics will synchronize to the transmitters' dynamics. In thus allowing the receiver the freedom of which dynamical system to implement, we enlarge the class of master/slave systems that allow synchronization. Note that at this point we do not consider the actual physical realization of the new receiver's dynamical system. In certain applications this may be crucial, but this aspect lies beyond the scope of the present paper, see however [20].

The problem just described is in fact the observer problem from control theory. For linear dynamical systems a complete solution to the problem is well known [13]. For nonlinear systems only partial results exist $[27,11,15,16,28]$.

Besides the master-slave perspective on synchronization another viewpoint is expressed in [22]. There synchronization is seen as the design of a (feedback) mechanism for the receiver, using the transmitted signal, so as to ensure that the controlled receiver synchronizes with the transmitter. This approach to synchronization is in essence a control problem, which we do not discuss in this paper, but see $[4,20]$.

A standard approach in solving the observer problem in control theory is to use as receiver a copy of the transmitter (of course with unknown initial state) modified with a term depending on the difference between the received signal and its prediction derived from the observer. The additional term aims at attenuating the difference between the state of the transmitter and the state of the observer system. This procedure may be shown to be successful in many instances, but certainly no global validity can be claimed. The synchronization 
problem requires one to establish global asymptotic stability for the zero solution of the error dynamics, i.e. the dynamics governing the difference between the transmitter state and observer state. Rigorous proofs often rely on Lyapunov arguments. Most of the existing results concerning synchronization also rely on Lyapunov based arguments $[9,7]$ and [12].

That a solution to the above synchronization problem, or observer problem, may be feasible under certain conditions may be deduced from the Takens embedding theorem [26], which is closely related to the observability property for nonlinear dynamical systems $[1,2]$. In essence the observability property states that the history of the transmitted signal contains all the information required to reconstruct a state variable for the master dynamics. The observability property is a generic property of dynamical systems. However it falls short of implying the existence of an observer or receiver that synchronizes. In the case of linear systems the link between observability (or better detectability) and the existence of an observer can be made explicitly. However in the nonlinear context the situation is not that clear, and apart from some local results, cited before, few results are available.

In [19] we have argued that the complexity of the transmitter dynamics is of little concern in the observer design or synchronization. Since synchronization of complex or chaotic dynamical systems is believed to be of utmost importance in practical secure communication this conclusion may seem little surprising. On the other hand we will argue in this paper that in the observer design of complex dynamical systems there may be room for exploiting chaos. Our results in this direction are partial, like is the case with the general nonlinear observer problem, and will be established by means of a number of examples from the synchronization literature. In a future publication a more complete picture will be presented.

Throughout the paper we use standard notation from nonlinear control, and we refer to $[21,19]$ for details. The organization of this note is as follows. In section 2 we discuss synchronization for the hyperchaotic Rössler system. In section 3 we treat the observer/synchronization problem for the Lorenz system and in section 4 we do the same for the Chua-circuit. Concluding remarks are given in section 5 .

\section{The hyperchaotic Rössler system}

Consider the hyperchaotic Rössler system (see [25])

$$
\left\{\begin{array}{l}
\dot{x}_{1}=-x_{2}-x_{3} \\
\dot{x}_{2}=x_{1}+0.25 x_{2}+x_{4} \\
\dot{x}_{3}=3.0+x_{1} x_{3} \\
\dot{x}_{4}=-0.5 x_{3}+0.05 x_{4}
\end{array}\right.
$$

together with the output equation

$$
y=x_{3} \text {. }
$$

Standard synchronization methods from [24] or [7] seemingly fail to achieve a proper synchronization mechanism. Also, a linearized observer as proposed in [25], will not always yield synchronization, see [25]. Following the methodology of $[19]$ we treat $(1,2)$ in a similar fashion as the usual Rössler system. In order to do so, we note that whenever (1) is initialized at a point with $x_{3}(0)>0$ then $x_{3}(t)>0$ for all $t>0$. This permits to introduce the coordinate change in stateand output-space

$$
\begin{aligned}
\left(z_{1}, z_{2}, z_{3}, z_{4}\right) & =\left(x_{1}, x_{2}, \ln x_{3}, x_{4}\right) \\
\tilde{y} & =\ln y .
\end{aligned}
$$

In these new coordinates, the system $(1,2)$ takes the form

$$
\left\{\begin{array}{c}
\dot{z}_{1}=-z_{2}-\exp \left(z_{3}\right) \\
\dot{z}_{2}=z_{1}+0.25 z_{2}+z_{4} \\
\dot{z}_{3}=z_{1}+3 \exp \left(-z_{3}\right) \\
\dot{z}_{4}=0.05 z_{4}-0.5 \exp \left(z_{3}\right) \\
\tilde{y}=z_{3}
\end{array}\right.
$$

Since the linear part of (5), (6) is observable, and since the only nonlinearities in (5) depend on the measured output it is clear that a simple observer can be constructed as

$$
\left\{\begin{array}{l}
\dot{\tilde{z}}_{1}=-\tilde{z}_{2}+k_{1}\left(\tilde{z}_{3}-z_{3}\right)-\exp \left(z_{3}\right) \\
\dot{\tilde{z}}_{2}=\tilde{z}_{1}+0.25 \tilde{z}_{2}+\tilde{z}_{4}+k_{2}\left(\tilde{z}_{3}-z_{3}\right) \\
\dot{\tilde{z}}_{3}=\tilde{z}_{1}+k_{3}\left(\tilde{z}_{3}-z_{3}\right)+3 \exp \left(-z_{3}\right) \\
\dot{\tilde{z}}_{4}=0.05 \tilde{z}_{4}+k_{4}\left(\tilde{z}_{3}-z_{3}\right)-0.5 \exp \left(z_{3}\right)
\end{array}\right.
$$

where the gains $k_{1}, k_{2}, k_{3}$ and $k_{4}$ have to be chosen appropriately. Note that the error dynamics under the coordinate changes (3) and (4) are linear. The above reasoning, which is purely based on the observation that the system admits linear observable error dynamics after suitable coordinate transformations, does not employ any knowledge on the complexity of the system (1). On the other hand, it is conjectured that the hyperchaotic Rössler possesses, like the Rössler system, a chaotic attractor and for appropriate initial conditions the system will remain confined to a bounded box $B$ in $\mathcal{R}^{4}$. Note that this conjecture, though natural from the way the hyperchaotic Rössler system was constructed, is far away from being proved. This box $B$ contains the chaotic attractor of (1) and is contained in the set $\left\{x \in \mathcal{R}^{4} \mid x_{3}>0\right\}$. the (local) observability of the system (1), (2) requires that the 4 functions $h(x), L_{f} h(x), L_{f}^{2} h(x), L_{f}^{3} h(x)$ are independent in $B$; here $f$ denotes the vectorfield defined in (1) and $h(x)=x_{3}$ is the output map defined in (2). In the present case we obtain the functions $x_{3}, 3.0+x_{1} x_{3},-x_{2} x_{3}-x_{3}^{2}+3.0 x_{1}+x_{1}^{2} x_{3},-x_{3} x_{4}+\ldots \ldots$. which are $\left(x_{3}>0\right.$ !) independent. But then, see [11], a 
high-gain observer exists for the system (1) restricted to the bounded region $B$ :

$$
\begin{cases}\dot{\tilde{x}}_{1}=-\tilde{x}_{2}-\tilde{x}_{3} & +k_{1}\left(\tilde{x}_{3}-x_{3}\right) \\ \dot{\tilde{x}}_{2}=\tilde{x}_{1}+0.25 \tilde{x}_{2}+\tilde{x}_{4} & +k_{2}\left(\tilde{x}_{3}-x_{3}\right) \\ \dot{\tilde{x}}_{3}=3.0+\tilde{x}_{1} \tilde{x}_{3} & +k_{3}\left(\tilde{x}_{3}-x_{3}\right) \\ \dot{\tilde{x}}_{4}=-0.5 \tilde{x}_{3}+0.05 \tilde{x}_{4} & +k_{4}\left(\tilde{x}_{3}-x_{3}\right)\end{cases}
$$

where $k_{1}, k_{2}, k_{3}, k_{4}$ are chosen sufficiently large, see [19]. Clearly, computationally the observer (11) might be preferable to the observer (10), where one first has to compute the appropriate coordinates although (11) is a suitable (high-gain) observer only if the functions $h(x), L_{f} h(x), L_{f}^{2} h(x)$ and $L_{f}^{3} h(x)$ are verified to be independent.

\section{The Lorenz system}

The well-known Lorenz system is given by the equations

$$
\left\{\begin{array}{l}
\dot{x}=\sigma(y-x) \\
\dot{y}=-x z+r x-y \\
\dot{z}=x y-b z
\end{array}\right.
$$

Together with the output signal

$$
w=x
$$

the transmitter $(9,10)$ - which exhibits chaotic motion for suitable parameters $\sigma, r$ and $b$-admits for synchronization the receiver

$$
\left\{\begin{aligned}
\dot{\tilde{x}} & =\sigma(\tilde{y}-\tilde{x}) \\
\dot{\tilde{y}} & =-x \tilde{z}+r x-\tilde{y} \\
\dot{\tilde{z}} & =x \tilde{y}-b \tilde{z}
\end{aligned}\right.
$$

The fact that (11) and (9) synchronize was discovered by Pecora and Carroll in [24] and has lead to the huge interest in synchronization. Introducing $e_{x}=$ $x-\tilde{x}, e_{y}=y-\tilde{y}$ and $e_{z}=z-\tilde{z}$ it follows that the error dynamics are given as

$$
\left\{\begin{array}{l}
\dot{e}_{x}=\sigma\left(e_{y}-e_{x}\right) \\
\dot{e}_{y}=-x e_{z}-\ell_{y} \\
\dot{e}_{z}=x e_{y}-b e_{z}
\end{array}\right.
$$

which can be shown to be asymptotically stable at $\left(e_{x}, e_{y}, e_{z}\right)=(0,0,0)$ by introducing the Lyapunov function

$$
V\left(e_{x}, e_{y}, e_{z}\right)=\frac{1}{2}\left(\frac{1}{\sigma} e_{x}^{2}+e_{y}^{2}+e_{z}^{2}\right)
$$

having as time-derivative along (12)

$$
\dot{V}\left(e_{x}, e_{y}, e_{z}\right)=-\left(e_{x}-\frac{1}{2} e_{y}\right)^{2}-\frac{3}{4} e_{y}^{2}-b e_{z}^{2} .
$$

On the other hand, a general format for an observer for $(9,10)$ is given by

$$
\left\{\begin{array}{rlr}
\dot{\tilde{x}} & =\sigma(\tilde{y}-\tilde{x}) & +k_{1}(\tilde{x}, \tilde{y}, \tilde{z}, x) \\
\dot{\tilde{y}}=-\tilde{x} \tilde{z}+r \tilde{x}-\tilde{y} & +k_{2}(\tilde{x}, \tilde{y}, \tilde{z}, x) \\
\dot{\tilde{z}}=\tilde{x} \tilde{y}-b \tilde{z} & +k_{3}(\tilde{x}, \tilde{y}, \tilde{z}, x)
\end{array}\right.
$$

with $k_{1}(\tilde{x}, \tilde{y}, \tilde{z}, z), k_{2}(\tilde{x}, \tilde{y}, \tilde{z}, x)$ and $k_{3}(\tilde{x}, \tilde{y}, \tilde{z}, x)$ suitable smooth functions that satisfy $k_{i}(x, \tilde{y}, \tilde{z}, x)=0$ for all $(x, \tilde{y}, \tilde{z})$. In fact, the particular receiver (11) is obtained by taking

$$
\left\{\begin{array}{l}
k_{1}(\tilde{x}, \tilde{y}, \tilde{z}, x)=\sigma(\tilde{x}-x) \\
k_{2}(\tilde{x}, \tilde{y}, \tilde{z}, x)=\tilde{x} \tilde{z}-x \tilde{z}+r(x-\tilde{x}) \\
k_{3}(\tilde{x}, \tilde{y}, \tilde{z}, x)=x \tilde{y}-\tilde{x} \tilde{y}
\end{array}\right.
$$

but one can seek a better error tracking by adding to (16) additional gains $\tilde{k}_{1}(\tilde{x}, \tilde{y}, \tilde{z}, x), k_{2}(\tilde{x}, \tilde{y}, \tilde{z}, x)$ and $\tilde{k}_{3}(\tilde{x}, \tilde{y}, \tilde{z}, x)$ that satisfy $\tilde{k}_{i}(x, \tilde{y}, \tilde{z}, x)=0$, for all $(x, \tilde{y}, \tilde{z})$.

Remark As noted in [19] an alternative to the receiver (11) consists in using the receiver as

$$
\left\{\begin{array}{l}
\dot{\tilde{y}}=-x \tilde{z}+r x-\tilde{y} \\
\dot{\tilde{z}}=x \tilde{y}-b \tilde{z}
\end{array}\right.
$$

and establishing that $(x, \tilde{y}, \tilde{z})$ converges to $(x, y, z)$ as $t$ goes to infinity. This follows easily by taking as Lyapunov function $\frac{1}{2}\left(e_{y}^{2}+e_{z}^{2}\right)$ for the $\left(e_{y}, e_{z}\right)$-dynamics. Clearly (17) corresponds to one particular reduced observer for the system $(9,10)$. The general structure for a reduced observer is slightly different, but similar to the general full order observer (15). Both in the reduced observer case and the full order observer the selection of the functions $\left(k_{i}(\tilde{x}, \tilde{y}, \tilde{z}, x), i=1,2,3\right.$, as to improve the error response is far from trivial, and certainly more difficult than in the linear case. We will leave this issue for future research.

An interesting alternative for the design of an observer (receiver) for the system $(9,10)$ lies in the use of an extended Kalman filter for $(9,10)$. Typically, in this case the signal (10) is replaced by a noisy one:

$$
w=x+\eta
$$

with $\eta \sim N(0, R)$ a white noise signal. The structure of the extended Kalman filter is given by

$$
\begin{cases}\dot{\hat{x}}=\sigma(\hat{y}-\hat{x}) & +k_{1}(t)(w-\hat{x}) \\ \dot{\hat{y}}=-\hat{z} \hat{x}+r \hat{x}-\hat{y} & +k_{2}(t)(w-\hat{x}) \\ \dot{\hat{z}}=\hat{y} \hat{x}-b \hat{z} & +k_{3}(t)(w-\hat{x})\end{cases}
$$

where the gain vector $k(t)=\left(k_{1}(t) k_{2}(t) k_{3}(t)\right)^{T}$ is given by

$$
k(t)=P(t)\left(\begin{array}{l}
1 \\
0 \\
0
\end{array}\right) R^{-1}
$$

with $P(t)$ the solution of a corresponding matrix Riccati differential equation

$$
\left\{\begin{aligned}
\dot{P} & =F(t) P+P F(t)^{T}-P H(t)^{T} R^{-1} H(t) P \\
P(0) & =P_{0}>0
\end{aligned}\right.
$$

where $F(t)=\frac{\partial f}{\partial x}(\hat{x}(t)), H(t)=\frac{\partial h}{\partial x}(\hat{x}(t))$, with $f$ and $h$ denoting the right hand side in $(9)$ resp. (10). 
At this point, no claim for good performance of the Extended Kalman filter is made. This is usual, but there may be reasons the filter may act (reasonably) well for the Lorenz system (9) which possesses a chaotic attractor. A Kalman filter type observer has been considered earlier, see [3] and references in there, but the fact that (9) when initialized within the domain of attraction of the chaotic attractor remains confined to a finite box, is helpful in the filter. Much work remains to be done here, but some preliminary simulations show the effectiveness of using a Kalman filter as observer. Especially, care should be taken for the 'design' parameters in the filter, i.e. $R, P_{0}$ and $\left(\hat{x}_{0}, \hat{y}_{0}, \hat{z}_{0}\right)$.

\section{The Chua circuit}

The Chua circuit is given by the equations

$$
\begin{aligned}
\frac{d}{d t}\left(\begin{array}{l}
x_{1}(t) \\
x_{2}(t) \\
x_{3}(t)
\end{array}\right) & =\left(\begin{array}{ccc}
-\frac{G}{G} & \frac{G}{C} & 0 \\
\frac{G}{Q} & -\frac{G}{Q} & \frac{1}{Q} \\
0 & -\frac{1}{L} & 0
\end{array}\right)\left(\begin{array}{l}
x_{1}(t) \\
x_{2}(t) \\
x_{3}(t)
\end{array}\right) \\
& +\left(\begin{array}{c}
-\frac{1}{C} f\left(x_{1}(t)\right) \\
0 \\
0
\end{array}\right), \\
& y(t)=\left(\begin{array}{lll}
1 & 0 & 0
\end{array}\right) x(t) .
\end{aligned}
$$

The nonlinearity $f$ in (25) is given by $f(y)=Q_{1} y+$ $1 / 2\left(Q_{2}-Q_{1}\right)\left(\left|y+y_{b}\right|-\left|y-y_{b}\right|\right)$ for some positive $Q_{1}$, $Q_{2}$ and $y_{b}$. This nonlinearity is not smooth, but this does not affect the discussion. Because $|f(y)| \leq \gamma|y|$ for some $\gamma>0$ is is easily shown that the solutions for all initial conditions are well defined on $\mathcal{R}^{3}$. Chua's circuit is shown to have a chaotic behavior for suitable chosen $Q_{1}, Q_{2}$ and $y_{b}$. Clearly the linear part of the system equations (25) is observable. An observer may thus be constructed as

$$
\begin{gathered}
\frac{d}{d t}\left(\begin{array}{c}
\hat{x}_{1}(t) \\
\hat{x}_{2}(t) \\
\hat{x}_{3}(t)
\end{array}\right)=\left(\begin{array}{ccc}
-\frac{G}{G} & \frac{G}{C} & 0 \\
\frac{G}{Q} & -\frac{G}{Q} & \frac{1}{Q} \\
0 & -\frac{1}{L} & 0
\end{array}\right)\left(\begin{array}{l}
x_{1}(t) \\
x_{2}(t) \\
x_{3}(t)
\end{array}\right) \\
+\left(\begin{array}{c}
-\frac{1}{C} f\left(x_{1}(t)\right) \\
0 \\
0
\end{array}\right)+\left(\begin{array}{c}
k_{1} \\
k_{2} \\
k_{3}
\end{array}\right)(\hat{y}(t)-y(t)) . \\
\hat{y}(t)=\left(\begin{array}{lll}
1 & 0 & 0
\end{array}\right) \hat{x}(t) .
\end{gathered}
$$

The choice $k_{1}=k_{2}=k_{3}=0$ will yield asymptotically stable linear error dynamics [6]. Using different gains allows us to select a faster error response. An interesting - and challenging - alternative for the output (23) in the Chua circuit is to take instead the output

$$
y=\left(\begin{array}{lll}
0 & 1 & 0
\end{array}\right) x
$$

It is clear that the synchronization problem for $(22,26)$ could be approached using the methods described in the previous sections. However, in [14] a particularly structured observer for $(22,26)$ is suggested, namely

$$
\begin{aligned}
\frac{d}{d t}\left(\begin{array}{c}
\hat{x}_{1} \\
\hat{x}_{2} \\
\hat{x}_{3}
\end{array}\right) & =\left(\begin{array}{ccc}
-\frac{G}{C} & \frac{G}{C} & 0 \\
\frac{G}{Q} & -\frac{G}{Q} & \frac{1}{Q} \\
0 & -\frac{1}{L} & 0
\end{array}\right)\left(\begin{array}{c}
\hat{x}_{1} \\
\hat{x}_{2} \\
\hat{x}_{3}
\end{array}\right) \\
& +\left(\begin{array}{c}
-\frac{1}{C} f\left(\hat{x}_{1}\right) \\
0 \\
0
\end{array}\right)+\left(\begin{array}{c}
0 \\
K\left(x_{2}-\hat{x}_{2}\right) \\
0
\end{array}\right)
\end{aligned}
$$

In [14] it is claimed that for $K$ sufficiently large, synchronization of (27) and (22) occurs. However, see also [18], it is not clear whether this is true for all possible initial states for (22) resp. (27). In fact, it is easy to see that if (22) starts at 0 and (27) not, no synchronization can occur, and the true question is whether $x=0$ is the only exception of this kind. Analysis and simulations on the error system $(22,27)$ - which is piecewise linear - clarifies that whenever $x_{1}$ enters the strip $-1<x_{1}<1_{1}$ then $\hat{x}_{1}$ grows. Since the system (22) possesses a chaotic attractor where $x_{1}$ traverses an interval $[-p, p]$ with $p>1$, it follows that 'many' trajectories for $(22)$ will induce the temporarily destabilizing effect for $\hat{x}_{1}$ in (27), but since the average time that $x_{1}$ is in the interval $(-1,1)$ is about $14 \%$ there is still good chance that for most trajectories synchronization exists. This leaves open whether this is the case for all trajectories.

\section{Concluding remarks}

The main lesson the reader could take from the examples of the previous sections is that synchronization of complex (chaotic) dynamic systems, or, equivalently, the observer problem for a complex (chaotic) system, may be succesfully solved by exploiting the chaotic nature of the system. Thus, not only standard observer properties are in this case important, but also the chaotic nature helps. Further work is needed as to see how systematic the given examples can be approached.

Acknowledgements In the preparation of this work the (simulation) study of Anders Robertsson (Lund) on the Chua system has been useful.

\section{References}

[1] D. Aeyels, Generic observability of differentiable systems, SIAM journal of Control and Optimization, Vol. 19, pp. 595-603, 1981. 
[2] D. Aeyels, On the number of samples necessary to achieve observability, Systems $\&$ Control Letters, Vol 1, pp. 92-94, 1981.

[3] G. Besancon, Contributions a l'etude et a l'observation des systemes non lineaire avec recour au calcul formel, $\mathrm{PhD}$ thesis, Institut National Polytechnique de Grenoble, 1996.

[4] I.I. Blekhman, A.L. Fradkov, H. Nijmeijer, A.Y. Pogromsky, On self-synchronisation and controlled synchronisation, Systems $\&$ Control Letters, to appear, 1997.

[5] T.L. Carroll, L.M. Pecora, Synchronizing chaotic circuits, IEEE Trans Circuits and Systems, Vol. 38, No. 4, pp. 453-456, 1991.

[6] Chai Wan Wu, L. Chua, A simple way to synchronize chaotic systems with applications to secure communication systems, International Journal of Bifurcation and Chaos, Vol. 3, No. 6, pp. 1619-1627, 1993.

[7] Chai Wan Wu, L. Chua, A unified framework for synchronization and control of dynamical systems, International Journal of Bifurcation and Chaos, Vol. 4, No. 4, pp. 979-998, 1994.

[8] G. Chen, Control and synchronization of chaos, a bibliography, Dept. of Electrical Engineering, University of Houston, TX, USA, available via ftp:uhoop.egr.uh.edu/pub/TeX/chaos.tex, 1997.

[9] K.M. Cuomo, A.V. Oppenheim, S.H. Strogratz, Synchronization of Lorenz-based chaotic circuits with application to communication, IEEE Trans Circuits and Systems II, Vol. 40, No. 10, pp. 626-633, 1993.

[10] M. Ding, E. Ott, Enhancing synchronisation of chaotic systems, Physical Review E, Vol. 49, No. 2, pp. 945-948, 1994.

[11] J. Gauthier, H. Hammouri, S. Othman, A simple observer for nonlinear systems, applications to bioreactors, IEEE Trans Aut. Cont., Vol. 37, No. 6, pp. 875-880, 1992.

[12] R. He, P. Vaidya, Analysis and synthesis of synchronous periodic and chaotic systems, Phys Rev A, Vol. 46, No. 12, pp. 7387-7392, 1992.

[13] T. Kailath, Linear System, Prentice Hall, 1980.

[14] T. Kapitaniak, Controlling chaos, theoretical and practical methods in nonlinear dynamics, Academic Press, London, 1996.

[15] A.J. Krener, A. Isidori, Linearization by output injection and nonlinear observers, Systems 89 Control Letters, Vol. 3, pp. 47-52, 1983.

[16] A. Krener, J. Respondek, Nonlinear observers with linearizable error dynamics, SIAM J. Control and Optimization, Vol. 23, pp. 47-52, 1985.

[17] O. Morgül, E. Solak, Observer based synchronisation of chaotic signals, Physical Review E, Vol. 54, No. 5, pp. 4803-4811, 1996.

[18] H. Nijmeijer, On chaotic observer design, Proc. Workshop on Open Problems in Mathematical Systems Theory and Control, (Eds. V. Blondel \& M. Vidyasagar), Univ. Liège, Belgium, pp. 55-57, 1997.
[19] H. Nijmeijer and I.M.Y. Mareels, An observer looks at synchronization, IEEE Transactions on Circuits and Systems I, to appear, 1997.

[20] H. Nijmeijer, I.M.Y. Mareels, On the controlled synchronization of dynamical systems, Proc. 2nd Asian Control Conference, Seoul, Korea, pp. 357-360, 1997.

[21] H. Nijmeijer, A. van der Schaft, Nonlinear Dynamical Control Systems, Springer Verlag, 1990.

[22] M.J. Ogorzalek, Taming chaos-part 1:synchronization, IEEE Transactions on Circuits and SystemsI Fundam. Theory and Applic. Vol. 40, pp. 693-699, 1993.

[23] E. Ott, T. Sauer and J.A. Yorke (eds.), Coping with chaos: analysis of chaotic data and the exploitation of chaotic systems, Wiley-Interscience Publ., New York, 1994.

[24] L.M. Pecora, T.L. Carroll, Synchronization in chaotic systems, Physical Review Letters, Vol. 64, No. 8, pp. 821-824, 1990.

[25] J.H. Peng, E.J. Ding, M. Ding and W. Yang, Synchronization hyperchaos with a scalar transmitted signal, Physical Rev. Lett., Vol. 76, no. 6, pp. 904-907, 1996.

[26] F. Takens, Detecting strange attractors in turbulence, Lecture Notes in Mathematics, Vol. 898, pp. 366-381, 1981.

[27] K. Tchon, H. Nijmeijer, On output linearization of observable dynamics, Control Theory and Advanced Technology, Vol. 9, No. 4, pp. 819-857, 1993.

[28] X.H. Xia, W.B. Gao, Nonlinear observer design by observer error linearization, SIAM Journal of Control and Optimization, Vol. 27, pp. 199-216, 1989. 